\title{
Risk factors for hospital admission of Brazilian children with non-rotavirus diarrhoea: a case control-study
}

\author{
Maria Yury T. Ichihara ${ }^{a, *}$, Laura C. Rodrigues ${ }^{b}$, Carlos A. S. T. Santos ${ }^{c, d}$, Maria da Glória L. C. Teixeira ${ }^{a}$ \\ and Mauricio L. Barreto ${ }^{a, d}$
}

\begin{abstract}
${ }^{a}$ Institute of Collective Health, Federal University of Bahia, Rua Basilio da Gama, s/n, Campus Universitário do Canela Salvador, CEP 40110-040, Salvador, Bahia, Brazil; ' London School of Hygiene \& Tropical Medicine, London, United Kingdom; 'State University of Feira de Santana, Bahia, Brazil; ' Gonçalo Muniz Research Center, FIOCRUZ, Salvador, Bahia, Brazil

*Corresponding author: Tel: + 5571 32837445; E-mail: my.ichihara@gmail.com
\end{abstract}

Received 10 December 2014; revised 29 April 2015; accepted 1 May 2015

\begin{abstract}
Background: Rotavirus has been the leading cause of severe cases of acute diarrhoea (AD) among children worldwide, however, in the same areas, a large reduction in AD related to rotavirus has been observed after the introduction of the rotavirus vaccine. In Brazil, where there is a high rotavirus vaccine coverage, AD caused by pathogens other than rotavirus is still a frequent cause of outpatient visits and hospitalizations among children under 5 years.
\end{abstract}

\begin{abstract}
Methods: A hospital-based case-control study enrolled children aged 4 to 24 months admitted to 10 hospitals from all five Brazilian Regions. Cases $(n=1178)$ were children admitted with diarrhoea who tested negative for rotavirus in a stool sample. Controls $(n=2515)$ were children admitted without diarrhoea, frequency matched to cases by sex and age group. We estimated odds ratios using logistic regression, in a hierarchical approach according to a previously defined conceptual framework. Population-attributable fractions (PAF) were estimated for each variable, each block and for all significant variables in the latter model adjusted.
\end{abstract}

\begin{abstract}
Results: The factors studied accounted for $41 \%$ of the non-rotavirus AD hospital admissions and the main risk factors included lack of adequate excreta disposal $(\mathrm{PAF}=12 \%)$, untreated drinking water $(\mathrm{PAF}=11 \%)$ and a history of previous hospitalization due to $\mathrm{AD}(\mathrm{PAF}=21 \%)$. Low socio-economic conditions, no public water supply, crowding and low weight-for-age made smaller contributions.

Conclusions: These findings further our knowledge of risk factors associated with severe $A D$ in the post-rotavirus vaccination era. We recommend further increase in coverage of basic sanitation, improvements in water quality and further expansion of primary healthcare coverage to reduce the occurrence of non-rotavirus severe diarrhoea and subsequent hospitalization of Brazilian children.
\end{abstract}

Keywords: Child hospitalization, Diarrhoea, Non-rotavirus diarrhea, Risk factors for diarrhea

\section{Introduction}

Diarrhoea remains a major global health problem and is a frequent cause of outpatient visits and hospitalizations. ${ }^{1}$ More than 2.5 billion cases of diarrhoea occur annually among children under 5 years of age, usually in the first 2 years of life. ${ }^{2}$

Our current understanding is that severe diarrhoea occurs among vulnerable populations in developing countries due to poor health, a lack of access to primary healthcare services and poor environmental conditions and hygiene at home. ${ }^{2}$ Several pathogens have been identified as putative causes in moderate-to-severe diarrhea in children: viruses, mainly rotavirus, but also norovirus, astrovirus and adenovirus; bacteria (mainly Escherichia coli, Shigella, Campylobacter, Salmonela) and protozoas (Giardia lambia, Entamoebahistolytica and Cryptosporidium). ${ }^{3-5}$

The causal network behind the occurrence of acute diarrhoea (AD) hospitalization varies according to the different etiologic agents involved and their routes of transmission and the living conditions of the population. ${ }^{2,6,7}$ Rotavirus of group A (RV-A) has been recognized as the leading cause of severe $A D$ in children in developed and in developing countries, and a major cause of death in poor countries. ${ }^{2}$ Worldwide, it has been reported to be responsible for almost 100 million episodes of $A D$ each year, and for $40 \%$ of all $A D$ hospitalizations in children under 5 years. ${ }^{1,2}$ However, after the introduction of the rotavirus vaccine, 
in particular in areas with high vaccine coverage, the role of this pathogen in the global burden of childhood AD morbidity, hospitalizations and mortality has been decreasing. ${ }^{8-10}$

In Brazil, before the introduction of the rotavirus vaccine, the main etiological pathogens associated with hospitalized diarrhoea were bacteria (e.g., E. coli, Shigella and Salmonella) ${ }^{11-13}$ and rotavirus. ${ }^{14}$ For rotavirus infections, the frequency of positive stool samples varied from 22 to $38 \%$ in hospitalized children. After the introduction of the rotavirus vaccine in 2006, as mandatory and free of charge in the National Immunization Program, ${ }^{9}$ the frequency of $A D$ hospital admission was almost halved in the last decade (120 000 cases in 2006 to 69000 cases in 2011) (DATASUS/SVS/Ministry of Health). Other non-specific measures such as improvements in access to water and sanitation, ${ }^{15}$ in nutrition, ${ }^{16}$ access to primary healthcare ${ }^{17}$ and cash transfer programs, ${ }^{18}$ observed in the same period, have contributed to the decrease in rotavirus $A D$, but also to a reduction in $A D$ caused by other pathogens. Nowadays, a high proportion of AD childhood hospitalization is caused by pathogens others than rotavirus.

For a long time, the study of severe and hospitalized AD was predominantly focused on rotavirus. In this study we aimed to identify relevant risk factors related to non-rotavirus AD hospital admissions in Brazilian children using a hierarchical approach. $^{19-22}$

\section{Materials and methods}

\section{Study design}

This was a hospital-based case-control study frequency matched by sex and age group. We invited 17 hospitals enrolled in the RV-A AD National Surveillance System to participate in the study, based on their large numbers of RV-A positive samples in 2007, adequate organization and data accessibility. After consultation and agreement of logistical arrangements with the local, State and National Health Surveillance Units and the Regional and National Reference Laboratories, 10 general hospitals located in five macro-regions of Brazil accepted to participate in the study. Children were admitted to these hospitals with a wide range of diseases and came from a large geographical catchment area.

\section{Participants}

Eligible children

Children were eligible if they were admitted for the first time during the study period in any of the selected hospitals, and were aged between 4 and 24 months.

Information of the eligible children was reviewed to exclude children who had any health conditions presumed to be associated with a risk of hospitalization (prematurity, low birth weight, immunodeficiency, gastrointestinal disease, e.g., diverticulitis, malformations or neoplasm), or general signs and symptoms, or infectious diseases. In addition, we excluded children who acquired diarrhea while in hospital or who had diarrhoea for more than 3 weeks before hospitalization, as they did not represent the population needed for the study. The remaining children were put in a pool from which we selected cases and controls. Cases were selected from children who had been hospitalized for diarrhoea and controls from children hospitalized for other illness and who were not excluded based on the exclusion criteria.

Additional exclusion criteria were set for cases. Children with diarrhea were excluded from being cases in the study if they stayed under 24 hours in hospital (for logistic reasons), had rotavirus in stools (we wanted to study risk factors for non-rotavirus diarrhea) or had an associated disease (we wanted to study risk factors for diarrhea primarily caused by an enteropathogen and not secondary to another disease [e.g., urinary infectious, infectious respiratory, disease, milk allergy]).

There was an additional step to ensure that cases and controls had roughly the same distribution of sex and age on admission (frequency matching), according to the different risk factors for AD hospital admission of children. To achieve this, in each hospital, controls were classified into six groups: for each sex, there were three age groups at admission (4-6 months, 7-11 months and 12-24 months). From these groups, controls were then selected to be included in the study at random reflecting the frequency of sex and age group of the cases (frequency matching). We used the 'sample' command of Stata version 11.0 (College Stattion, TX, USA).

Cases were therefore children aged 4 to 24 months hospitalized for $A D$ (defined as three or more liquid stools in 24 hours, up to 14 days before admission), and who did not meet any of the exclusion criteria. Controls were children aged 4 to 24 months, admitted to the same hospital as the cases, with no exclusion criteria, and frequency matched to cases.

A minimum sample size of 576 cases and 2303 controls were necessary to observe an odds ratio of at least 1.4 for risk factors and a frequency of $10 \%$ of exposed controls at a $95 \%$ confidence interval, a power of $80 \%$ and a 1:4 exposed/unexposed ratio using EPI-INFO 7.0. A sample size of 1178 cases and 2515 controls was obtained in this study.

\section{Laboratory investigation of RV-A}

A single stool sample was collected up to 48 hours after admission, as part of the guidelines of the AD RV-A Surveillance System and the General Coordination of Public Laboratories of the Ministry of Health of Brazil. Rotavirus was initially investigated by enzyme immunoassay (EIA) using commercial kits (Dako or Oxoide) and confirmed by EIA and polyacrylamide gel, according to Leite et al. ${ }^{23}$ Details of these methods are published elsewhere. ${ }^{24}$

\section{Data collection}

Interviewers were selected and trained by the study investigators and professionals in charge of the rotavirus AD surveillance in each hospital. These same professionals supervised data collection from July 2008 to August 2011. Cases (non-rotavirus AD) and controls were defined based on information from a questionnaire answered by the child's career, related to the child, the family and the household (identification, clinical history and evolution, socio-economic status, sanitation, water supply and frequency, breastfeeding and the nutritional status of the child and maternal factors) and from the stool examinations done at the reference laboratory, which identified positive stool samples for rotavirus and its genotypes. All the information from the questionnaires was revised and data was assessed for completeness and consistency. 


\section{Study variables}

A conceptual framework was developed including variables with potential to be associated with $A D$ hospitalization, taking into account the inter-relationships among them (Figure 1). ${ }^{19,21}$ The variables potentially co-linear (family income, type of housing, maternal education, alcohol consumption during pregnancy, characteristics of the toilet and bathroom used by the family and the number of visits to health services), even if significantly associated with hospital admission, were not included in the conceptual framework. Also, birthweight and exclusive breastfeeding were excluded because of the strong association with preterm birth, which is one of the major risk factors for hospitalization among children (proximal causes).

We measured the individual household socio-economic position using the 'ownership of goods'. Goods in this context are household possessions (refrigerator, freezer, stove, television, car, telephone, washing machine, microwave, computer and dishwasher). Given the complexity and variations in this form of wealth, ownership of goods is often used as a measure of socioeconomic condition in low- and middle-income countries. We used 'more than four goods' as a cut off point for good socioeconomic status to make this comparable to other studies from Brazil and Latin America. ${ }^{21}$

Variables were grouped into six blocks, according to their interrelationships with the $A D$ hospital admission (Figure 1): the distal level had socio-economic and demographic variables, including family and mother's conditions (Block 1). At the intermediate level we defined three blocks: two related to the pathways of AD transmission (Block 2: sanitation and Block 3: contact) and one related to the maternal factors that affects the child's health (Block 4: maternal condition). The variables at this level were designed to mediate the effect of socio-economic and demographic factors or to be influenced by the effect of the variables from the proximal level or to have a direct effect on AD hospitalization. At the proximal level, the Blocks 5 (nutrition) and 6 (previous morbidity), had variables associated with susceptibility of children developing severe AD, which could have been influenced by the earlier levels or had a direct effect in AD hospital admission. This allowed us to estimate the overall and direct effect of the variables.

\section{Statistical analysis}

Different statistical approaches were used to explore complex dynamics inter-relationships between the many risk factors, and confusingly more than one approach is called hierarchical analysis. The hierarchical analysis we used here has been often used to explore prosed causal pathways, including pathogen routes of transmission diarrhea, and it is detailed elsewhere. ${ }^{19-22}$ In brief, it applies an effect-decomposition strategy that is based, in a step-by-step process, on the blocks of potential risk factors defined in the conceptual framework previously conceived based on our understanding of the mechanism of disease transmission.

Initially, we selected the variables with $p \leq 0.05$ at bivariate analysis. These variables were included in the logistic regression intra-block analysis. In each block, we identified variables that were significantly associated with $A D$ hospitalization $(p \leq 0.05)$ and they were included in the analysis according to the conceptual framework of risk factors, which takes into account the inter-relationships among them (Figure 1). The distal level variables were included first (Block1) (Model A), followed by variables in Blocks 2-4 of the intermediate level (Model B) and subsequently by variables in Blocks 5-6 of the proximal level (Model C). The variables remained in the models, when $p \leq 0.05$, using the Wald test, after adjustment for variables from the superior and the same levels. All the analyses were adjusted for age group and sex (frequency matching) and to take into account the correlation due the hospitals (clusters), we used the estimation of robust variance (generalized estimating equations). For the analysis we used the software STATA, version 12.0 (College Station, TX, USA).

This hierarchical approach enabled us to verify the role of a complex set of risk factors in non-rotavirus AD hospitalization. The intra-block analysis estimated the association between variables of each block and AD hospital admission. In the analysis of the distal level, Model A showed the effect of socio-economic status (block 1). With Model B, including significant variables of block 1 and all variables of Blocks 2-4, it was possible to observe the overall effect of the variables of Block 2-4 and the effect of socioeconomic variables in Block 1 not mediated by the variables in these three blocks. Model $C$, including significant variables of the Blocks 1-3 (variables of block 4 were not significant) and the variables of the blocks 5-6, estimated the overall effect of the blocks $5-6$, the effect of socio-economic condition not mediated by these two blocks and the effect not mediated by variables of Blocks 2-3.

In addition, assuming risk factors as independent effects, ${ }^{25}$ we estimated the population-attributable fractions (PAFs) using the formula: [(\% of exposed cases) x (OR-1)/OR]. We used the odds ratios (ORs) estimated in intra-block analysis to calculate PAFs for each risk factor. The summary PAFs for each block of variables was the result of the complements of the product of the complements of individual PAFs of the variables in the blocks. An overall summary PAF was calculated with the complements of the product of the complements of PAFs using OR's estimated in Model $C$. This represented the proportion of AD hospital admissions due to all the variables in the latter model. ${ }^{21,25}$

\section{Results}

A total of 4333 eligible children, aged 4 to 24 months, were recruited for the study from July 2008 to August 2011 . The eligible children ( $n=1440$ admitted with diarrhoea) had their stool samples tested for rotavirus by EIA, PAGE and RT-PCR. A total of 232 children with rotavirus-positive samples were excluded and 1178 cases were selected for this analysis. From a total of 2893 eligible children as controls (without diarrhoea), 2515 were selected by the frequency matching method (Figure 2).

The mean age of the cases and controls was 13 months. The characteristics of the study population and OR's (95\% CI) of the association between risk factors and $A D$ hospital admissions are shown in the Supplementary table. On average, cases had lower socio-economic and sanitary conditions. Their families had fewer than four goods (41.3\% [486/1178]), a large number of people were living in crowded households (17.3\% [204/1178] had 3 or more persons per room) and they were more likely to live in households with inadequate sanitation: (19.4\% [228/1178] had no public water supply; $60.5 \%$ [713/1178] had no connection to the 


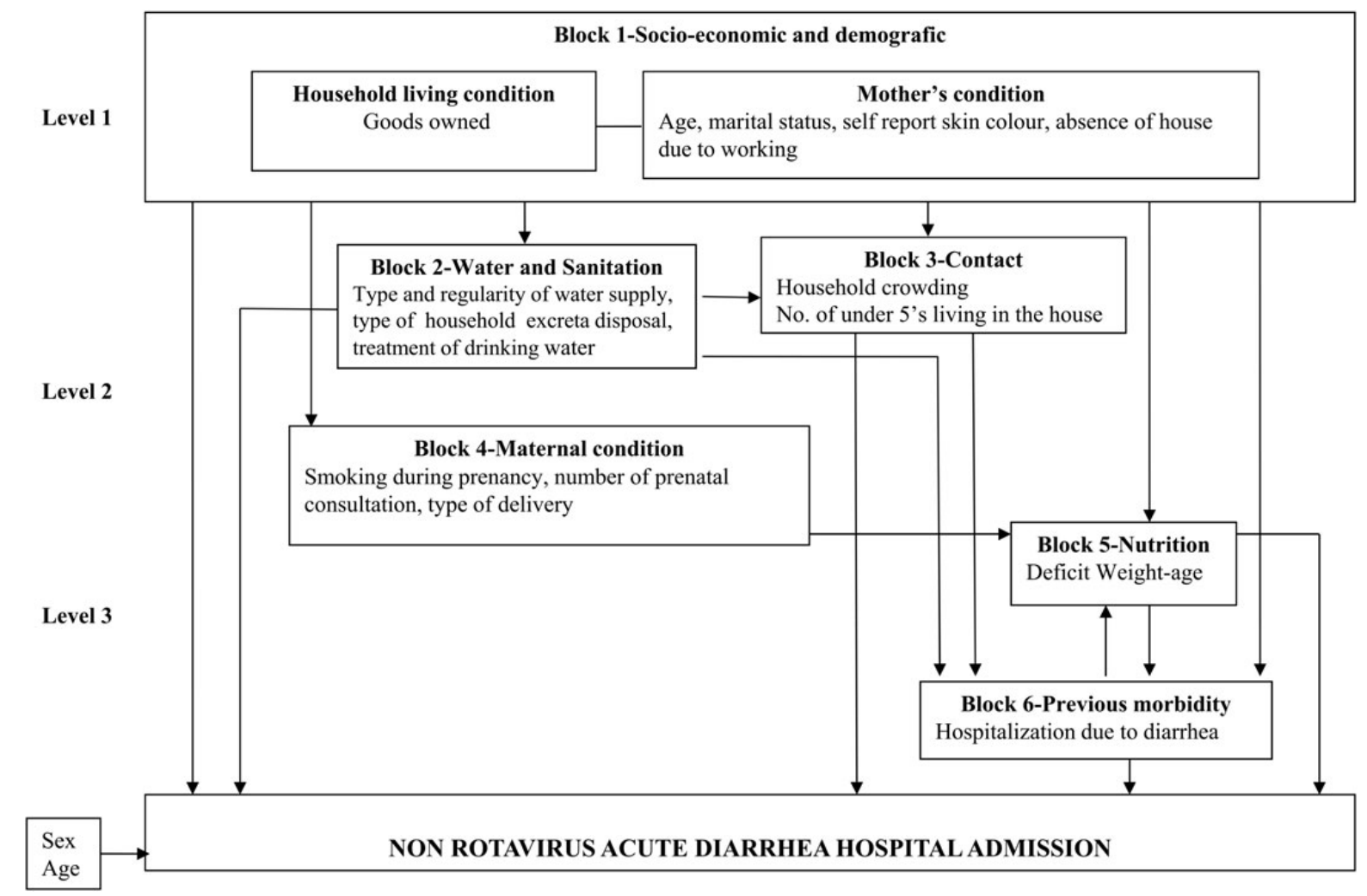

Figure 1. Conceptual framework with inter-relationships between risk factors and non-rotavirus acute diarrhoea hospital admission of Brazilian children.

public sewage system, $40.3 \%$ [475/1178] had no treatment of drinking water). Also, low weight-for-age was more common in cases $(12.7 \%-147 / 1178)$ than in the controls $(8.2 \%-201 / 2515)$ and a previous $A D$ hospitalization was six times more frequent in cases $(24.4 \%-288 / 1178)$ than in controls $(3.9 \%-97 / 2515)$. There was no difference in the mother's demographic characteristics and maternal factors between cases and controls.

The risk factors associated with $A D$ hospital admission were (see Supplementary table): ownership of fewer than four goods (OR 1.17; 95\% CI 1.01-1.34); no public water supply (OR 1.42; 95\% CI 1.19-1.71), no public household excreta disposal (OR $1.41 ; 95 \%$ CI 1.22-1.62), no treatment of drinking water (OR $1.41 ; 95 \%$ CI 1.22-1.62), three or more persons/room living in the household (OR 1.40; 95\% CI 1.16-1.70), deficit in weight-forage (OR 1.66; 95\% CI 1.33-2.09) and one or more previous AD hospitalization to the current admission (OR 8.13; 95\% CI 6.38-10.36).

Table 1 shows the odds ratios estimated for the intra-block analysis and for distal, intermediate and proximal levels for the risk factors: Model A (analysis of 1-distal level), Model B (analysis of 1-distal and 2-intermediate levels) and Model C (analysis of 1-distal, 2-intermediate and 3-proximal levels).

In the intra-block analysis, the following factors were significantly associated with AD hospital admission: ownership of fewer than four goods (Block 1: socio-economic factors); no piped public water supply, no connection to the public sewage system and no adequate treatment of drinking water (Block 3: sanitation); crowding (three or more persons per room) (Block
4: contact); deficit of weight-age (Block 5: nutrition); and had had one or more previous hospitalizations due to diarrhoea (Block 6: previous morbidity).

In Model A, ownership of fewer than four goods (Block 1: socioeconomic factors) was significantly associated with AD hospital admission in the distal level. In Model B, all factors related to sanitation remained significantly associated with AD hospitalization when the socio-economic variable was included in the analysis. Ownership of fewer than four goods (socio-economic factor) reduced the OR from 1.17 (Model A) to 1.04 (Model B). In addition, household crowding (meaning level of interpersonal contact) showed no statistical significance.

Model $\mathrm{C}$ showed the effect of variables defined as the proximal level on the occurrence of AD hospitalization. Deficit of weight-age and one or more previous AD hospitalizations remained significant when we introduced the variables from the distal and intermediate levels in the model. Furthermore, it presented the unmediated effects of sanitation and of socio-economic conditions. In this model, the socio-economic factor (goods ownership) was no longer statistically significant when sanitation factors were introduced in the model and these remained significantly associated with non-rotavirus AD hospital admission, even in the presence of the proximal level variables.

All factors in Model C explained $41 \%$ of the non-rotavirus AD hospital admissions in Brazilian children. A large proportion of PAF (26\%) was from Block 2: sanitation, mainly inadequate sanitation $(\mathrm{PAF}=13 \%)$ and treatment of drinking water $(\mathrm{PAF}=11 \%)$; and from Block 6: previous AD hospitalization ( $\mathrm{PAF}=21 \%$ ). 


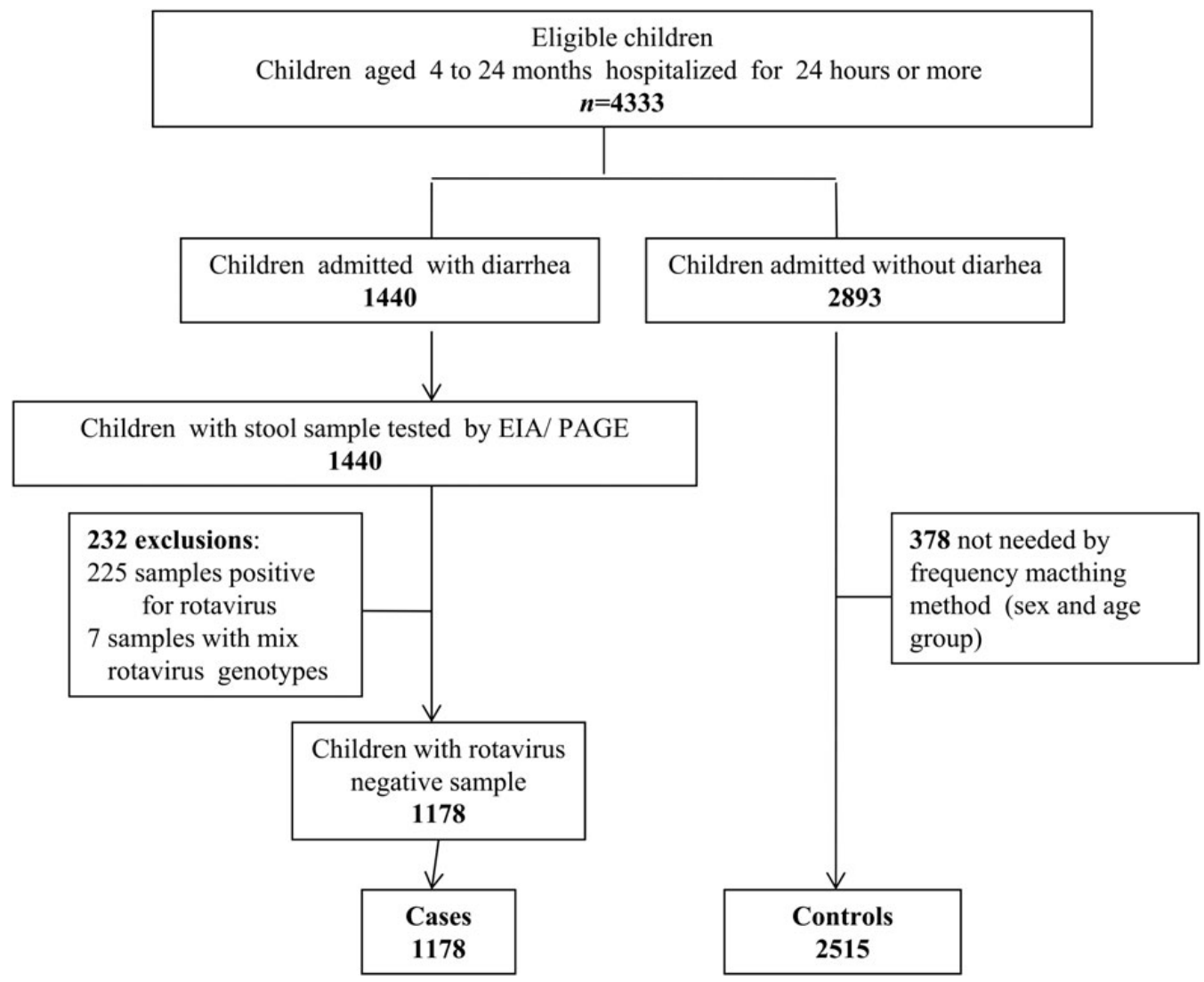

Figure 2. Study population of eligible children in Brazil.

\section{Discussion}

This study showed that $41 \%$ of non-rotavirus AD hospitalization of Brazilian children under 2 years was attributable to lower socioeconomic conditions, inadequate water supply and sanitation, low weight-for-age and one or more previous admissions for AD.

The effect of socio-economic factors was mediated by sanitation factors, which remained significant when we included the proximal variables in the model.

Our findings are consistent with social and environmental improvements in Brazil in recent years. ${ }^{26}$ In $2011,94 \%$ of Brazilian municipalities had piped water supplies, however, this may include households with intermittent water access, without connection to the public system and collecting water from a public tap. Only 55.2\% of municipalities had a public sewage system, showing great deficiencies in access to sanitation in the country. A Brazilian study showed that a $10 \%$ or more increase in combined water supply and sanitation coverage was associated with a $6 \%$ reduction in all AD hospitalization of children up to 5 years of age. ${ }^{27}$ Existing evidences suggest that in developing countries improvements in water quality reduced the risk of diarrhoea in young children by $17 \%$ and point-of-use quality water interventions reduced this by $15 \% .^{28}$

AD caused by enteropathogens (others than rotavirus), in general, has been associated to poverty, inadequate sanitation and water supply (quantity and quality) and precarious hygiene; while the magnitude of the risks observed varied among countries and regions. ${ }^{2,15,28-30}$ It has been estimated that in developing countries combined prevention strategies (improvement in access, quantity and quality of water, adequate sanitation and good hygiene practices within domestic and community settings) ${ }^{10}$ could reduce diarrhoea morbidity by $20-40 \%{ }^{28-30}$

There is a major difference in the pre- and post-vaccination for rotavirus era in Brazil: a large proportion of severe AD cases were caused by rotavirus before the introduction of the rotavirus vaccination, which has an inter-personal transmission route, therefore the role of deficiencies in sanitation and water supply were less pronounced. After the introduction of rotavirus vaccine, our study showed that over $80 \%$ of children admitted to hospital with $A D$ were due to non-rotavirus diarrhoea, caused by a large group of pathogens with transmission routes that are more dependent on sanitation and water supply. This implies that we have an epidemiologic situation where severe $A D$, despite its reduction, is again being caused by pathogens with transmission routes that dominated in the period pre-rotavirus vaccination. This finding is important since, very often, episodes of severe or hospitalized diarrhoea do not have an etiologic diagnosis in low- and middle-income settings.

We found that low weight-for-age accounted for a small proportion of non-rotavirus AD hospitalization. There is evidence of improvement in the nutritional status of Brazilian children ${ }^{16}$ and of a decrease in AD hospitalization as a result of strategies to fight poverty in Brazil (e.g., conditional cash transfers through the Bolsa Familia Program-BFP). ${ }^{18}$

It has been widely reported in the literature that accessibility to health services, exclusive breastfeeding, child immunization, 
Table 1. Odds ratios and 95\% confidence interval estimated from intra-block and hierarchical analysis of risk factors for non-rotavirus acute diarrhea hospital admission of Brazilian children up to 2 years, 2008 to 2011

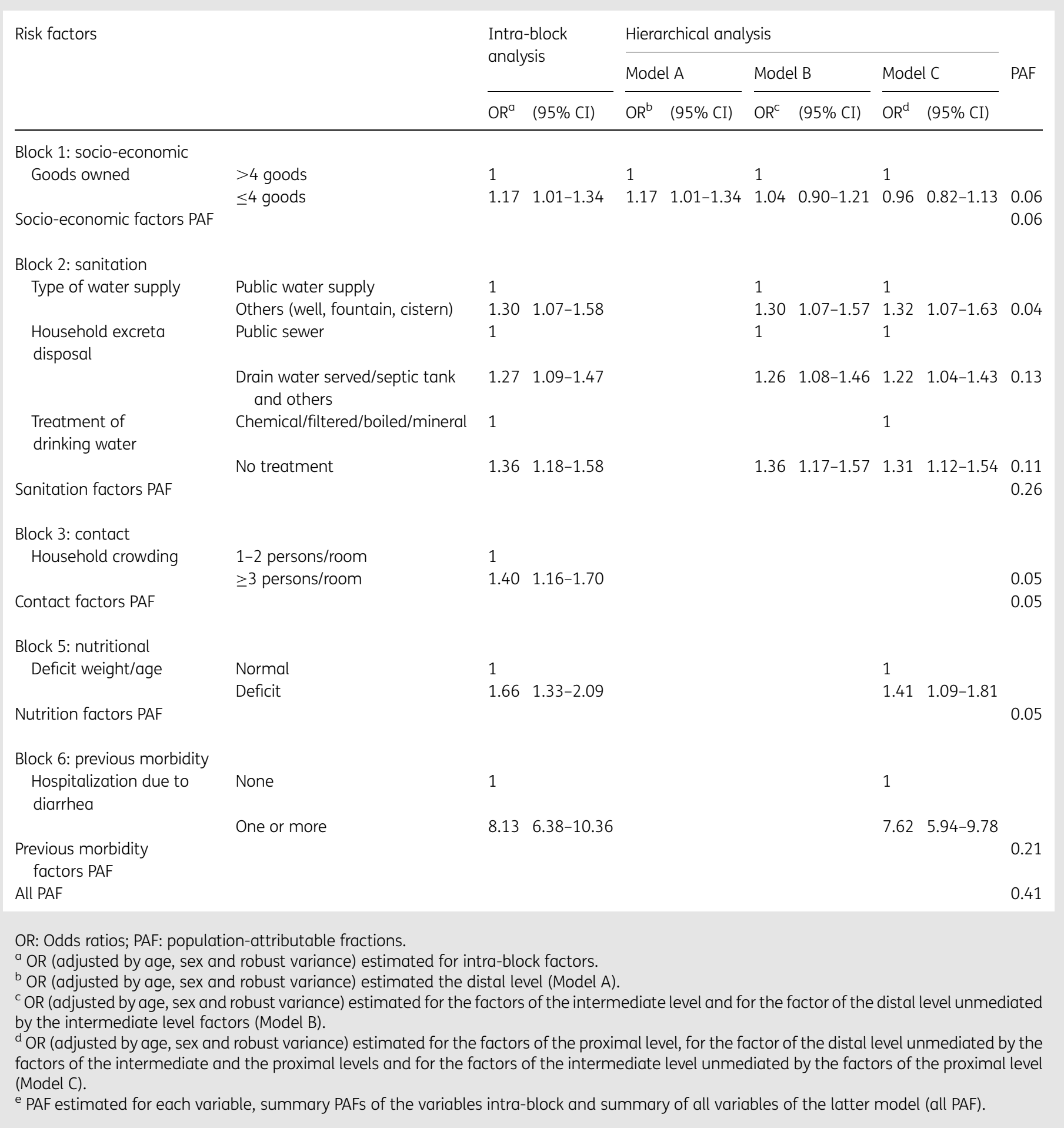

monitoring of growth and development in children under 5 years and early treatment of acute diarrhoea could reduce severe diarrhoea, repeated AD hospitalization and death. ${ }^{31}$
In the last two decades, Brazil has promoted an expansion of access to primary healthcare through the Family Health Program (FHP), which has achieved an estimated population coverage of 
54\% (Ministry of Health of Brazil/Basic Attention Department and IBGE). There is evidence that the increased access to primary healthcare has reduced the rate of $A D$ hospitalization among Brazilian children. ${ }^{17}$ Previous AD hospitalization contributed 21\% of the AD hospital admission of children under 2 years.

Finally, diarrhoea results from many causative etiologic agents with different routes of transmission (water, sanitation, food contamination and person-to-person). Hospitalization can be prevented by access to primary care, which can identify episodes of diarrhoea and give adequate oral rehydration, avoiding the severe outcome. Because of these complex inter-relationships, geographical comparisons are not necessarily useful other than to indicate the potential for further reductions. This study is a secondary analysis of data generated to evaluate rotavirus vaccine effectiveness. We did not know all the causative agents for the cases reported in this study, similar to the routine of clinical practice in the health services. ${ }^{13,32}$ However, our results are relevant as they identify the risk factors associated with severe AD and are useful to help guide health policy to define strategies to reduce $A D$ hospitalizations in the post- rotavirus vaccination era.

This study has some limitations. We did not collect information on some potential risk factors for severe $A D$, such as additional environmental factors (i.e., garbage, quantity and quality of water, drainage wastewater, whether access to piped water was inside the household), community and personal hygiene (i.e., food preparation and child excreta disposal) and father's education and maternal behavior at home. In addition, information on sanitation were obtained from interviews with caregivers or guardians of the children, which could have a recall bias. The level of child dehydration on hospital admission was also not verified and no adjustment was done to the weight registered on the medical records, which could overestimate the risk associated with 'deficit weight-for-age'. However, the children were usually rehydrated before admission (in the emergency department) and the body fluid volume was probably reconstituted before they were weighted on admission to the ward.

One of the strengths of the study was the hierarchical approach (informed by a conceptual framework) that considered the inter-relationship of the variables and allowed us to identify different pathways that contributed to the occurrence of diarrhoea. This enabled the identification of inadequate sanitation as a contributor to low socio-economic conditions; and to show that sanitation and one or more previous $A D$ hospitalization were the main factors associated with non-rotavirus AD hospital admission.

\section{Conclusions}

Based on these results we recommend further increases in basic sanitation coverage, improvements in water quality and further expansion of primary healthcare to reduce the occurrence of severe non-rotavirus diarrhoea and subsequent hospitalization of Brazilian children.

In spite of rotavirus vaccination, diarrhoea caused by others pathogens remains a major health problem for Brazilian children. Policy makers should take note that other essential measures like expanding public sewage and treatment of drinking water, alongside rotavirus vaccination, need to be taken into account in order to reduce hospitalization by diarrhoea. It is necessary to continue to investigate the changing determinants of severe diarrhoea in children, using a dynamic approach based on integrative conceptual models. ${ }^{33}$ The results should support strategies of prevention and intervention to reduce mortality and morbidity due to diarrhoea and its consequences for nutrition and child development.

\section{Supplementary data}

Supplementary data are available at Transactions Online (http:// trstmh.oxfordjournals.org/).

Authors' disclaimer: The Health Surveillance of Ministry of Health of Brazil supported the study but played no role in the study design, in collection, analysis and interpretation of data, or in the writing of the report, nor in the decision to submit the article for publication.

Authors' contributions: MYTI designed the study, managed the field work, analysed and interpreted the data and wrote the first draft of the paper; CASTS, LCR and MdaGLCT contributed to analysis and interpretation of the data; MLB conceived the study, contributed to the study design, analysis and interpretation of the data. All authors read and approved the final manuscript. MYTI is the guarantor of the paper.

Funding: This work was supported by Health Surveillance of Ministry of Health of Brazil.

\section{Competing interests: None declared.}

Ethical approval: This study was approved by the Committee of Institute of Collective Health, Federal University of Bahia [Protocol 017-08/CEP/ ISC-2008].

\section{References}

1 Glass RI, Bresee JS, Turcios R et al. Rotavirus vaccines: targeting the developing world. J Infect Dis 2005;192(Supp1):S160-70.

2 UNICEF/WHO. Diarrhoea: why children are still dying and what can be done. New York: United Nations International Children's Emergency Fund; 2009.

3 Kollof KL, Nataro JP, Blacwelder WC et al. Burden and aetiology of diarhoeal disease in infants and young children in developing countries (the Global Multicenter Study, GEMS): a prospective, case-control study. Lancet 2013;382:209-22.

4 Walker CL, Rudan I, Liu L et al. Global burden of childhood pneumonia and diarrhoea. Lancet 2013;381:1405-16.

5 Clasen T, Roberts I, Rabie Tet al. Interventions to improve water quality for preventing diarrhea. Cochrane Database Syst Rev 2006;19: CD004794.

6 Patel K, Thillainayagam. Diarrhoea. Medicine 2008;37:23-7.

7 Cairncross S, Blumenthal U, Kolsky P et al. The public and domestic domains in the transmission of disease. Trop Med Int Health 1996;1:27-34.

8 Desai R, Oliveira LH, Parashar UD et al. Reduction in morbidity and mortality from childhood diarrhoeal disease after species A rotavirus vaccine introduction in Latin America- a review. Mem Inst Oswaldo Cruz 2011;106:907-11.

9 do Carmo GM, Yen C, Cortes J et al. Decline in diarrhoea mortality and admissions after routine childhood rotavirus immunization in Brazil: a time-series analysis. PLoS Med 2011;8:e1001024. 
10 Yen C, Armero Guardado JA, Alberto P et al. Decline in rotavirus hospitalizations and health care visits for childhood diarrhoea following rotavirus vaccination in El Salvador. Pediatr Infect Dis J 2011;30(1 Suppl):S6-S10.

11 Diniz-Santos DR, Santana JS, Barretto JR et al. Epidemiological and microbiological aspects of acute bacterial diarrhea in children from Salvador, Bahia, Brazil. Braz J Infect Dis 2005;9:77-83.

12 Cruz CB, de Souza MC, Serra PT et al. Virulence Factors Associated with Pediatric Shigellosis in Brazilian Amazon. BioMed Res Int 2014;2014:539697.

13 Moura MRS, de AL, Mello MJG, Calábria WB et al. The frequency of Escherichia coli and its sensitivity to antimicrobials in children aged under five years admitted to hospital for treatment of acute diarrhea. Rev Bras Saúde Matern Infant 2012;12:173-82.

14 Gurgel RQ, Cunliffe NA, Nakagomi O, Cuevas LE. Rotavirus genotypes circulating in Brazil before national rotavirus vaccination: a review. J Clin Virol 2008;43:1-8.

15 Barreto ML, Genser B, Strina A et al. Effect of city-wide sanitation programme on reduction in rate of childhood diarrhoea in Northeast Brazil: assessment by two cohort studies. Lancet 2007;370:1622-8.

16 Paes-Sousa R, Santos LMP, Miazaki ES. Effect of conditional cash transfer programme on child nutrition in Brazil. Bull World Health Organ 2011;89:496-503.

17 Rasella D, Aquino R, Barreto ML. Reducing childhood mortality from diarrhea and lower respiratory tract infections in Brazil. Pediatrics 2010;126:e534-40.

18 Rasella D, Aquino R, Santos CA et al. Effect of a conditional cash transfer programme on childhood mortality: a nationwide analysis of Brazilian municipalities. Lancet 2013;382:57-64.

19 Victora CG, Huttly SR, Fuchs SC, Olinto MT. The role of conceptual frameworks in epidemiological analysis: a hierarchical approach. Int J Epidemiol 1997;25:224-7.

20 Fucs SS, Victora CG, Fachel J. Hierarchical model: a proposal for a model to be applied in the investigation of risk factors for severe diarrhea [in Portugese]. Rev Saúde Pública 1996;30:168-78.

21 Ferrer SR, Strina A, Jesus SR et al. A hierarchical model for studying risk factors for childhood diarrhoea: a case-control study in a middle-income country. Int J Epidem 2008;37:805-15.
22 Genser B, Strina A, Teles CA et al. Risk factors for childhood diarrhoea incidence: dynamic analysis of a longitudinal study. Epidemiology 2006;17:658-67.

23 Leite JP, Alfieri AA, Woods PA et al. Rotavirus $G$ and P types circulating in Brazil: characterization by RT-PCR, probe hybridization, and sequence analysis. Arch Virol 1996;141:2365-74.

24 Ichihara MYT, Rodrigues LC, Santos CAST et al. Effectiveness of rotavirus vaccine against hospitalized rotavirus diarrhoea: A case-control study. Vaccine 2014;32:2740-7.

25 Bruzzi P, Green SB, Byar DP et al. Estimating the population attributable risk for multiple risk factors using case-control data. Am J Epidemiol 1985;122:904-14.

26 Ministério do Planejamento, Orçamento e Gestão. IBGE. Atlas de saneamento; 2011.

27 Rasella D. Impact of the water for all program (PAT) on childhood morbidity and mortality from diarrhea in the Bahia State, Brazil. Cad Saúde Pub 2013;29:40-50.

28 Cairncross S, Hunt C, Boisson S et al. Water, sanitation and hygiene for the prevention of diarrhoea. Int J of Epidemiol 2010;39(Supp 1):i193-i205.

29 Esrey RA, Feachem RG, Hughes JM. Interventions for the control of diarrhoeal diseases among young children: improving water supplies and excreta disposal facilities: Bull World Health Organ 1985;63:757-72.

30 Fewtrell L, Kaufmann RB, Kay D et al. Water, sanitation, and hygiene interventions to reduce diarrhea in less developed countries: a systematic review and meta-analysis. Lancet Infect Dis 2005;5:42-52.

31 Macinto J, Guanais FC, de Fatima M, de Souza M. Evaluation of the impact of the Family Health Program on infant mortality in Brazil, 1990-2002. J Epidemiol Community Health 2006;60:13-9.

32 Croxen MA, Law RJ, Scholz R et al. Recent advances in understanding enteric pathogenic Escherichia coli. Clin Microbiol Rev 2013;26:822-80.

33 Eisenberg JN, Trostle J, Sorensen RJ, Shields KF. Toward a systems approach to enteric pathogen transmission: from individual independence to community interdependence. Annu Rev Public Health 2012;33:239-57. 